\title{
Adverse Events after Inappropriate In-Home Ultraviolet Phototherapy: Sunburn and Exacerbation of Psoriasis
}

\author{
Jong Seo Park, Jungyoon Moon, Seong Jin Jo \\ Department of Dermatology, Seoul National University College of Medicine, Seoul, Korea
}

\section{Dear Editor:}

Ultraviolet (UV) phototherapy is one of the effective treatment options of psoriasis. In-home UV phototherapy has advantage of avoiding the inconvenience of frequent hospital visits ${ }^{1}$. However, in Korea, it is not prescribed by physicians because of unreliable phototherapy devices and poor patient compliance. Here, we describe a case of sunburn and exacerbation of psoriasis, adverse events caused by the inappropriate in-home UV phototherapy. The study was approved by the Institutional Review Board of the Seoul National University Hospital (IRB no. $\mathrm{H}$ 1709-112-888). We received the patient's consent form about publishing all photographic materials.

A 57-year-old woman with a 5-year history of psoriasis visited the outpatient clinic with multiple rectangular patches on her abdomen, back, and thigh. The rectangular lesions were symmetric, well-demarcated, dark-colored, uniform, and about $10 \times 5 \mathrm{~cm}$ in size. Well-demarcated erythematous plaques with silvery scales had developed recently, overlying the dark, rectangular patches on the thigh (Fig. 1). The patient reported that she had used an in-home UV phototherapy device (Wavelength: 315 $\mathrm{nm} \pm 10 \%$, Irradiance: $1.7 \mathrm{~mW} / \mathrm{cm}^{2} \pm 10 \%$ ) without consulting her dermatologist. She was unaware of the precautions of phototherapy, so she had used the device for over two minutes, twice as long as recommended by the manufacturer. After the second treatment, she had burn injuries on her abdomen, back, and thigh. Given her medi-
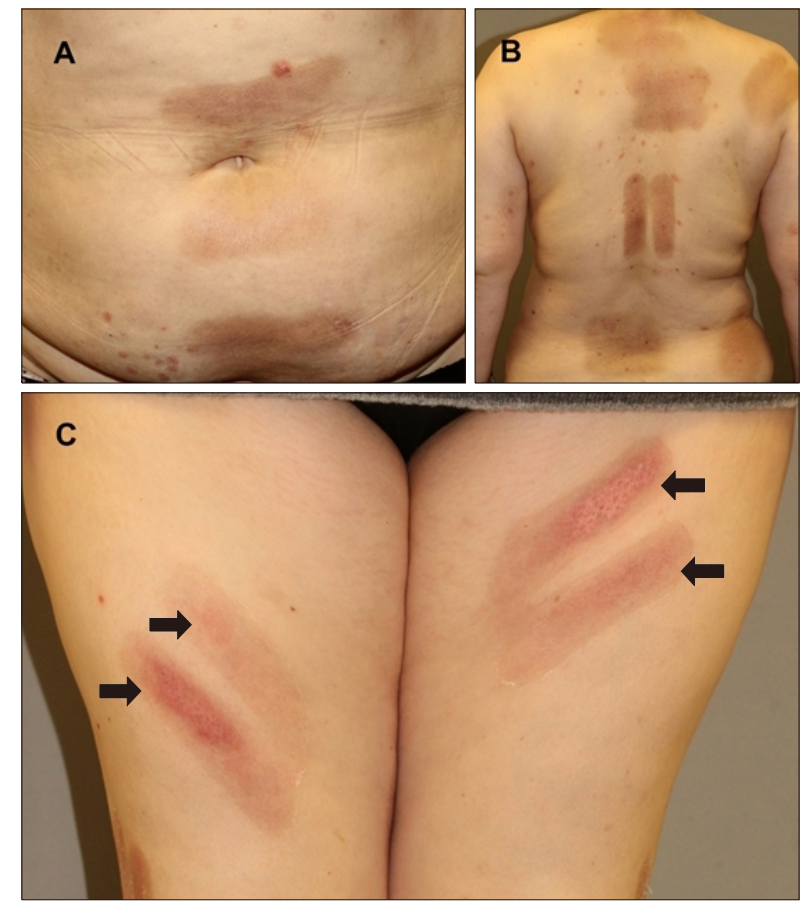

Fig. 1. Clinical pictures at the patient's first visit to our clinic. She presented with multiple symmetric, well-demarcated, darkcolored rectangular patches and some erythematous plaques with silvery scales. We diagnosed her with post-inflammatory hyperpigmentation on the (A) abdomen, (B) back, and exacerbation of psoriasis after sunburn (black arrows) on the (C) thigh after inappropriate use of in-home ultraviolet phototherapy.

Received November 20, 2017, Revised January 3, 2018, Accepted for publication February 5, 2018

Corresponding author: Seong Jin Jo, Department of Dermatology, Seoul National University College of Medicine, 101 Daehak-ro, Jongno-gu, Seoul 03080, Korea. Tel: 82-2-2072-2417, Fax: 82-2-742-7344, E-mail: sj.jo@snu.ac.kr ORCID: https://orcid.org/0000-0002-2501-7672

This is an Open Access article distributed under the terms of the Creative Commons Attribution Non-Commercial License (http://creativecommons.org/ licenses/by-nc/4.0) which permits unrestricted non-commercial use, distribution, and reproduction in any medium, provided the original work is properly cited.

Copyright (C) The Korean Dermatological Association and The Korean Society for Investigative Dermatology 


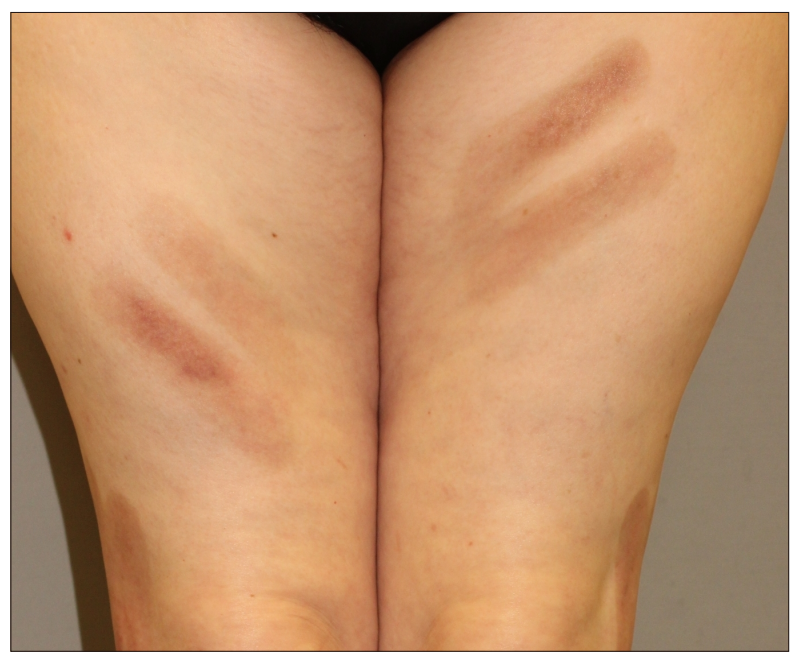

Fig. 2. Improvement of psoriatic lesions on the thigh after a month of treatment with a topical vitamin D analogue and a steroid.

cal history, we diagnosed the factitious rectangular lesions as post-inflammatory hyperpigmentation and the erythematous scaly plaques as exacerbation of psoriasis after sunburn. The psoriatic lesions were controlled within a month using topical treatment with a vitamin $\mathrm{D}$ analogue and a steroid. The patient was instructed to abstain from using in-home phototherapy (Fig. 2).

The patient's exacerbated psoriatic lesions can be explained by the Koebner phenomenon, defined as the appearance of new skin lesions on areas of cutaneous injury. Burning trauma is one of the causes of the Koebner phenomenon. They are observed in a variety of dermatological diseases, including psoriasis.

Phototherapy is a specialized treatment, and when treated inappropriately like in our case, it causes side effects instead of treatment effects. For safety and efficacy, the frequency and intensity of UV phototherapy should be decided by specialized dermatologists. However, in Korea, in-home phototherapy devices can be easily purchased via online shopping without prescription and can be used indiscriminately without the guidance of a doctor. Although some studies introduced in-home UV phototherapy as an economical, convenient, and efficacious way of treating psoriasis $^{1-3}$, easy accessibility of the in-home device without a doctor's prescription is accompanied with high risk of adverse events.

Here, we report a case of adverse events due to improper use of in-home UV treatment device. For safe and effective treatment in Korea, we recommend that the purchase and use of in-home UV phototherapy devices should be managed through medical system and allowed only with a prescription from a dermatologist.

\section{CONFLICTS OF INTEREST}

The authors have nothing to disclose.

\section{ORCID}

Jong Seo Park, https://orcid.org/0000-0002-9662-0970

Jungyoon Moon, https://orcid.org/0000-0002-7575-0063

Seong Jin Jo, https://orcid.org/0000-0002-2501-7672

\section{REFERENCES}

1. Bhutani T, Liao W. A practical approach to home UVB phototherapy for the treatment of generalized psoriasis. Pract Dermatol 2010;7:31-35.

2. Koek MB, Buskens $E$, van Weelden $H$, Steegmans $P H$, Bruijnzeel-Koomen CA, Sigurdsson V. Home versus outpatient ultraviolet B phototherapy for mild to severe psoriasis: pragmatic multicentre randomised controlled non-inferiority trial (PLUTO study). BMJ 2009;338:b1542.

3. Eleftheriadou V, Ezzedine K. Portable home phototherapy for vitiligo. Clin Dermatol 2016;34:603-606. 